\title{
Kernos
}

Revue internationale et pluridisciplinaire de religion grecque antique

$15 \mid 2002$

Varia

Un buste parlant d'Épicure, un passage de Lucien ( Alexandre le faux prophète, 26) : la philosophie au péril des religions

\section{Renée Piettre}

\section{(2) OpenEdition}

Journals

Édition électronique

URL : http://journals.openedition.org/kernos/1371

DOI : 10.4000/kernos. 1371

ISSN : 2034-7871

Éditeur

Centre international d'étude de la religion grecque antique

Édition imprimée

Date de publication : 1 janvier 2002

ISSN : 0776-3824

Référence électronique

Renée Piettre, « Un buste parlant d'Épicure, un passage de Lucien (Alexandre le faux prophète, 26) : la philosophie au péril des religions », Kernos [En ligne], 15 | 2002, mis en ligne le 21 avril 2011, consulté le 20 avril 2019. URL : http://journals.openedition.org/kernos/1371 ; DOI : 10.4000/kernos.1371 


\section{Un buste parlant d'Épicure, un passage de Lucien (Alexandre le faux prophète, 26) : la phillosophie au périll des religions}

A quoi pouvait bien servir un buste d'Épicure converti en tête oraculaire? C'est la question que se posa Frederik Poulsen ${ }^{1}$, lorsque la restauration d'un superbe buste du fondateur du Jardin, réplique datée du I $^{\text {er }}$ siècle ap. J.-C. de l'original $\mathrm{du} \mathrm{III}^{\mathrm{e}}$ siècle, et de provenance inconnue ${ }^{2}$, lui révéla une cavité maladroitement creusée dans la base du buste et aboutissant à une perforation de la bouche susceptible de produire, moyennant l'introduction d'un tuyau dans l'orifice et le truchement d'un souffleur, l'illusion d'une tête parlante. Les étudiants de Poulsen avaient même tenté l'expérience et constaté la voix caverneuse produite par ce dispositif ${ }^{3}$. Or le percement avait abîmé le buste et ne pouvait donc pas s'expliquer par la destination première de l'objet. Par ailleurs, tous les exemples antiques et byzantins de statues animées, dont l'auteur fournit une riche moisson, relèvent de supercheries de prêtres. Poulsen concluait qu'on ne pouvait rendre compte de ce buste étrange que par l'hypothèse de son réemploi non épicurien, excluant l'attribution de ces pratiques illusionnistes aux Épicuriens eux-mêmes.

La découverte comme le dossier réuni restent d'un extrême intérêt. Mais pourquoi exclure d'emblée l'hypothèse qu'il aurait fallu examiner d'abord: existait-il la moindre chance que des Épicuriens se donnent à eux-mêmes le spectacle d'un portrait animé de leur maître et fondateur? Or, cette chance existe, si mince soit-elle en regard de toutes les réutilisations possibles d'un buste de philosophe.

1 Fr. Poulsen, "Talking, Weeping and Bleeding Sculptures. A chapter of the History of Religious Fraud", AArcb 16 (1945), p. 178-195.

2 Ny Carlsberg Glyptotek de Copenhague (I.N. 607). Acheté en 1890 à Rome chez Martinetti. Cf. F. Johansen, Greek Portraits, Copenhague, 1992, n 38 p. 96; K. Schefold, Die Bildnisse der antiken Dichter, Redner und Denker, Basel, 1943, p. 210; V. POuLSEN, Les poitraits grecs, Copenhague, 1954, p. 59 sq. et pl. XXIV, fig. 33; G.M.A. Richter, The Portraits of the Greeks, London, 1965, II, fig. 1178-1179.

3 Un buste parlant plutôt que vomissant flammes, eau ou fumée, dont Poulsen n'a pas vu la trace. 
On a beaucoup écrit sur les portraits épicuriens dans les mondes hellénistique et romain ${ }^{4}$. Bernard Frischer ${ }^{5}$ en explique la prolifération par des pratiques de recrutement et d'endoctrinement: les Épicuriens s'engageaient mutuellement à devenir semblables aux dieux ${ }^{6}$, en suivant le modèle d'Épicure lui-même; et le modèle se présentant à l'esprit sous la forme des simulacres qui en émanent, ils croyaient ne pouvoir mieux faire que de se mettre continûment sous les yeux les traits mêmes du maître, comme le résumé le plus direct de sa doctrine dont la leçon était ainsi soutenue par l'image ${ }^{7}$ autant que par les maximes répétées par coeur, le recueil de lettres lu et relu ${ }^{8}$, les points de doctrine constamment révisés dans la pleine franchise et la libre expression du doute qui étaient de règle au cours des entretiens amicaux de la secte ${ }^{9}$. La découverte à Autun d'une « anthologie épicurienne » sur un pavement de mosaïque, avec les portraits d'Épicure, de Métrodore et d'Anacréon accompagnés de citations invitant au plaisir ${ }^{10}$, peut, puisqu'il semble bien qu'il s'agisse d'un peu plus que d'un ornement de salle de banquet $^{11}$, illustrer la même fonction mnémotechnique et psychagogique de l'image, en liaison directe avec le texte, qui pourrait être placé dans la bouche

4. Cf. aussi V. Kruse-Berdoldt, Kopienkritiscbe Untersuchungen zu den Porträts des Epikur, Metrodor und Hermarch, Diss. Göttingen, 1975, p. 19, E13; H. Wrede, "Bildnisse Epikureischer Philosophen", MDAI(A) 97 (1982), p. 235-245 (p. 242); R. WINkEs, "The portraiture of Epikouros", RALouvain 16 (1983), p. 86; H. v. HeINTzE, in N. BoNACASA, A. DI VITA (éds), Studi in onore di Acbille Adriani, Roma, $1992^{2}$ [1984], III, p. 765 sq.; B. Schmaltz, MarbWPr (1985), p. 55; R. voN DEN HoFf, Pbilosopbenporträts des Frïb- und Hochbellenismus, München, 1994; P. Zanker, Die Maske des Socrates. Das Bild des Intellektuellen in der antiken Kunst, München \& The Regent University of California, 1995.

5 B. Frischer, The Sculpted Word, Epicureanism and Pbilosopbical Recruitment in Ancient Greece, Berkeley, Univ, of California P, 1982.

6 D.L., X, 135. Cf. R. Piettre, "Épicure, dieu et image de dieu : une autarcie extatique", RHR 216/1 (1999), p. 5-30; J. WARREN, "Epicurean Immortality", OSAPb (2000), p. 231-261.

7 Cic., De fin. V, 3 : Nec tamen Epicuri licet obliuisci, si cupiam, cuius imaginem non modo in tabulis nostri familiares, sed etiam in poculis et in anulis babent; PurNe, N.H. XXXV, 5 : Epicuri uoltus per cubicula gestant ac circumferunt secum.

8 D.L., X, 12; 35; 84.

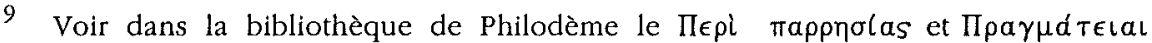
(C. Militello, Filodemo. Memorie Epicuree (PHerc. 1418 e 310). La scuola di Epicuro, Napoli, Bibliopolis, 1997. Et les analyses de N. DE WITT, Epicurus and bis Pbilosopby, Minneapolis, 1954; P. RAввоW, Seelenfübrung. Metbodik der Exerzitien in der Antike, München, 1954; P. НADot, Études de pbilosophie ancienne, Paris, Les Belles Lettres, 1998.

10 M. Blanchard-Lemée, A. Blanchard, "Épicure dans une anthologie sur mosaïque à Autun", CRAI (1993), p. 969-984; A. BlanCHARD, "Épicure 'Sentence Vaticane' 14 : Épicure ou Métrodore?", REG 104 (1991), p. 394-409; cf. dans le catalogue d'exposition de la ville d'Autun, Métrodore. Un philosopbe, une mosaïque. Musée Rolin, 6 juillet-30 septembre 1992, les contributions d'A. Blanchard, p. 49-54, et de M. Blanchard-Lemée, p. 55-57.

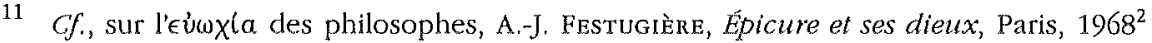
[1946], p. 34 sq., n. 3. 
des personnages, comme des phylactères de bande dessinée. Une manière équivalente de procéder a pu consister dans le recours à une image parlante. On sait que les Épicuriens ne reculaient devant aucun sacrifice pour se procurer des images suggestives. Plutarque les accuse d'acheter à n'importe quel prix la représentation de cet épisode clef de la vie d'Épicure qu'est la proscynèse de Colotès ${ }^{12}$. L'activité dialogique était si essentielle dans la représentation de la vie philosophique, que l'on rencontre l'exemple d'un groupe de quatre statuettes de marbre, trouvées dans les ruines d'une riche villa de Dion en Macédoine, figurant des personnages différenciés par leurs traits, mais tous drapés et assis dans la pose caractéristique d'Épicure, et comme en conversation ${ }^{13}$.

Il serait absurde d'en déduire que les Épicuriens auraient cru posséder la scène elle-même à travers son image animée, et la présence réelle de ses acteurs par le truchement de leurs portraits. Ils n'en considéraient pas moins ces images avec sérieux : toutes les perceptions nous sont apportées par des

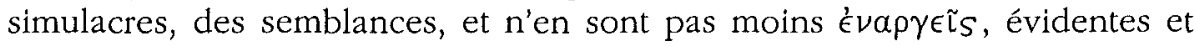
dignes de foi. Le problème est de les considérer en elles-mêmes, comme des images, et de tirer de ces images le renseignement exact, mais rien de plus, qu'elles peuvent apporter sur l'objet même dont elles émanent ${ }^{14}$. Toute la doctrine épicurienne de la perception des dieux paraît reposer précisément sur cette restriction, qui équivaut à un recueillement sur l'image ${ }^{15}$. Et cette image, comme le rappellent les fragments «Sur la forme du dieu » attribués à

12 Plut., Contre Colotès, 1117. Cf. R. Piettre, "La proscynèse de Colotès", Lalies 18 (1998), p. 185-202.

13 Non publié. Voir en attendant D. Pandermalis, Ergon (1987), p. 64 sq., fig. 64-65; BCH 111 (1988), p. 646, fig. 71; ArchRep 35 (1988-89), p. 66, fig. 92. Cf. Von den HofF, o.c. (n. 4), p. 70, no 7-10; Zanker, o.c. (n. 4), p. 229 sq. Il n'est pas certain, dit Zanker, qu'il s'agisse d'Épicure : mais peut-être du portrait idéalisé de citoyens honorables (deux sont imberbes : mais leurs têtes plus petites peuvent ne pas être originales), en un temps où la paideia dit le rang social. Un groupe de cinq philosophes dont deux affectent la pose d'Épicure a été trouvé à Athènes (G. Dontas, "Eıxovıбтıka B'", $A D$ 26A [1971], p. 16-33, pl. 1-8). Ces groupes d'intellectuels dialoguant ne sont pas spécifiques aux Épicuriens, $c f$. dans ZANker, o.c., p. 172, fig. 91, l'exèdre monumentale du Serapeion de Memphis, avec statues assises de poètes et philosophes (in ${ }^{\mathrm{e}}$ s. av. J.-C.), ou p. 310 fig. 167, une mosaíque d'Apamée figurant six philosophes, et Socrate au milieu, assis en demi-cercle ( $362 / 3$ ap. J.C.).

14 Théorie des simulacres : $c f$. D.L., X, 46-59; Lucr., III : Diog. CEN., fr. 9-10 et 15 Smith.

15 Citons K. Kleve, Gnosis Theon, SO Suppl. XIX, 1963; J. Bollack, La pensée du plaisir, Paris, 1975, commentaires à Mén.. 123-124, à KD 1 et scholie; J. KANY-Turpin, "Les images divines. Cicéron lecteur d'Épicure", RPbilos 1 (1986), p. 39-58; J. Moreau, "Épicure et la physique des dieux", REA 70 (1968), p. 286-294; D. LEMKe, Die Theologie Epikurs. Versuch einer Rekonstruktion, München, 1973 (Zetemata 57); A.A. Long, D.N. SEDLEY, The Hellenistic Philosopbers, Cambridge U.P., 1987, p. 129-149 (vol. I) et 143-154 (vol. II); J. MANSFELd, "Aspects of Epicurean Theology", Mnemosyne 46 (1993), p. 172-210; D. Oвbink, Philodemus On Piety I, Oxford U.P., 1996, passim. 
Démétrius Lacon (PHerc. 1055) ${ }^{16}$, a nécessairement forme humaine : si bien qu'on ne peut mieux approcher les dieux qu'en regardant l'homme qui leur ressemble le plus, à savoir Épicure ${ }^{17}$. Les Épicuriens invitent toujours leurs adeptes non à se détourner des images comme de pures illusions, mais au contraire à regarder de près les images mêmes, fussent-elles fantômes ou rêves, comme dans la lettre $\hat{A}$ la mère ${ }^{18}$, ou bien images des dieux. Si ces images sont considérées en tant qu'images, et non comme la chose même, au lieu d'épouvanter elles rassurent. Si je considère de même les statues des dieux comme la traduction plastique de la prolepse qui les définit comme des vivants indestructibles et bienheureux, je ne m'épouvanterai plus de leur gigantisme ni de la foudre qu'elles brandissent, mais je me réjouirai de la force et du bonheur qu'elles dénotent. Si je réfléchis que le buste d'Épicure montre les traits vénérés de la figure du maître, je me féliciterai de cet accès facile qu'il me donne à l'appel à la vie sereine. Toutes les images méritent d'être examinées avec attention ${ }^{19}$.

Mais le bonheur des dieux que l'Épicurien propose d'imiter sera plus accessible si au lieu de sculpter des statues terrifiantes on montre "les dieux joyeux et souriants ", comme le propose encore Diogène d'CEnoanda ${ }^{20}$. Certaines images en effet réalisent si parfaitement l'idéal de tranquillité (que l'examen soigneux des perceptions a pour vocation d'éviter de troubler), que leur seule vue apaise et réjouit. C'est alors une médecine bienfaisante que d'en proposer la multiplication, de manière que les yeux des hommes, les reconnaissant d'emblée, puissent constamment se reposer sur elles. Comme l'image de Dionysos se révèle au fond de la coupe du buveur une fois que l'ayant vidée il s'est enivré ${ }^{21}$, ainsi la sagesse d'Épicure apparaît à Memmius quand il s'assimile le breuvage amer des enseignements de Lucrèce. La douce contemplation des traits du maître aurait la même vertu pédagogique que le miel poétique dont Lucrèce enduit les bords de sa coupe. "Il nous faut faire

16 M. Santoro, (Demetrio Laconel, lLa forma del Diol (PHerc, 1055), Napoli, Bibliopolis, 2000.

17 Quel que soit le mode de perception, de la chose même je ne peux savoir plus que ces deux vérités générales : elle ne m'est accessible que par ses simulacres, de quelque profondeur qu'ils émanent; elle est formée d'une combinaison d'atomes qu'assembla et que désassemblera le hasard.

18 Diog. En., fr. 125-126 Smith.

$19 C f$. l'esthétique de son exposé par Philodème: le son n'a pas en lui-même des qualités éthiques ni motrices, l'âme n'est mise en mouvement que par la pensée contenue dans les mots du poème. La pensée doit toujours s'ajuster à chaque perception particulière, pour l'évaluer. Voir R. Janko, D. Armstrong, D. Delattrre dans C. Auvray-Assayas, D. Delattre (éds), Cicéron el Pbilodème. La polémique en pbilosopbie, Paris, Presses de l'E.N.S., 2001 (Études de littérature ancienne, 12), p. 283-296, p. 297-309, p. 371-384.

20 Diog. EN., fr. 19 Smith.

21 Cf. F. Lissarague, Un flot d'images. Une estbétique du banquet grec, Paris, 1987, fig. 94 et 107. 
le choix d'un homme de bien et l'avoir constamment devant les yeux de manière à vivre comme sous son regard. "22.

Diogène avait trouvé un autre véhicule immédiat de la doctrine : inscrire l'enseignement épicurien sur le mur d'un grand portique édifié à ses frais, de manière à ce que les yeux déchiffrent et que la mémoire assimile même involontairement les remedia d'Épicure. La disposition même des textes, la taille, le format variés des caractères en fonction de la hauteur du regard, anticipent en quelque sorte la mise en page ciblée de nos tabloïds.

Les procédés destinés à soutenir l'effort vers l'ataraxie prétendent non éblouir, mais réjouir. Ils s'opposent au spectaculaire, qui veut étonner, comme ils s'opposent à l'illusionnisme, qui prétend non seulement étonner (ainsi le théâtre étonne sans déguiser sa nature fictive), mais encore abuser.

Mais ne risque-t-on pas, à trop aplanir l'accès à la doctrine, à en adoucir l'ascèse, de séduire plus que de convaincre? Le poème de Lucrèce et l'inscription de Diogène relèvent déjà d'un tour de force. Avec la fabrication d'une tête parlante, qui rappelle la tête voyageuse d'Orphée ou les sortilèges de la craniomancie, ne bascule-t-on pas dans l'illusionnisme?

Les élèves de Poulsen s'étaient amusés à faire parler le buste d'Épicure d'une voix caverneuse, à travers un tuyau de bronze. Or Poulsen notait que la réfection de l'orifice entre les lèvres de la statue avait permis de produire une diction bien plus nette. On peut donc penser que le dispositif était destiné non à impressionner, mais à proférer clairement les enseignements fondamentaux du Jardin. Le tuyau a pu être fait non de bronze, mais de trachées de grues, ou de tout autre oiseau à long cou, dont il conviendrait de mettre à l'épreuve les qualités acoustiques: outre un exemple d'idole parlante chez Lucien (infra), on en rencontre l'emploi dans des pratiques magiques $^{23}$, où cependant les signes écrits prennent la place de l'oral. Dans le cas d'Épicure, on peut imaginer que des pratiques éprouvées aient pu servir, sans qu'il y eût la moindre volonté de tromper, à « animer » de la propre parole du maître des traits si puissamment rendus par le ciseau.

22 SÉN., Ep. XI, 8 = Us. Epicurea, fr. 210.

23 Preisendanz, PGM I V 370 sq., p. 194 : on fabrique une figurine d'Hermès en pâte,

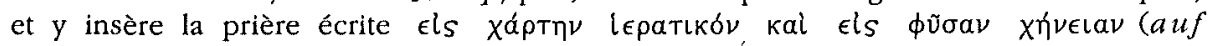
biëratiscbes Papier oder auf die Luftröbre einer Ganś, traduit Preisendanz, mais le kai pourrait indiquer que le papier était enroulé dans la trachée, puis celle-ci insérée dans la

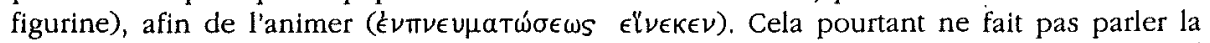
statue ni ne suffit à produire l'oracle, comme l'indique la suite de la recette. PGM III, 296

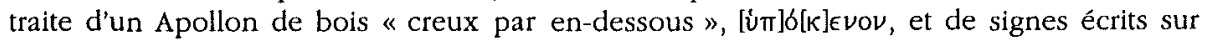
une feuille, qu'il n'est cependant pas question d'insérer dans la figurine. Apulée disposait d'une statuette de Mercure à qui il disait rendre des dévotions conformes aux prescriptions de Platon, mais qu'on le soupçonnait d'utiliser à des fins magiques (Apol., 61-65). 
Les Épicuriens n'ont pas toujours parlé d'une seule voix ${ }^{24}$ : jusqu'à quel point la fidélité au maître pouvait-elle entraîner un culte non seulement de sa mémoire, mais de sa présence vivante à travers sa parole et ses disciples assemblés pour lui ressembler? Jusqu'à quel point l'objectif de l'unique salut à diffuser auprès des amis du Kèpos autorise-t-il l'utilisation d'appâts aimablement attrayants? Le recours à la poésie chez Lucrèce est-il une trahison (le sens ainsi enveloppé sera-t-il moins obvie ?) ${ }^{25}$ ? La traduction latine vaut-elle l'original dans la langue des dieux qu'est le grec ${ }^{26}$ ? Tout le problème est dans le paradoxe épicurien de la vérité des apparences. Les simulacres sont vrais et convaincants, sources de toute connaissance : mais à condition d'être reconnus comme tels. Quels sont alors les meilleurs simulacres? Ceux qui illusionnent le plus (qui font oublier leur nature de simulacres) ou ceux qui se dénoncent eux-mêmes comme des copies? Les Épicuriens, comme Platon ${ }^{27}$, paraissent avoir opté pour les seconds. Nous suggérons qu'affecter une voix à la statue, loin d'ajouter à l'illusion, pouvait souligner le caractère artificiel de l'objet en le redoublant par l'artificieux. L'illusionniste aurait alors fait coup double : il aurait joint le texte à l'image, mais il aurait aussi introduit la touche d'invraisemblance qui trahissait l'image comme telle, tout en confiant à la parole l'énoncé de la doctrine vraie.

Une seconde hypothèse pèserait sans doute plus lourd, sans contredire nos précédentes analyses : c'est que nous ayons affaire à une caricature des pratiques épicuriennes. La persécution anti-épicurienne a pu utiliser contre la secte ses propres armes. En Crète, selon un témoignage d'Élien ${ }^{28}$, les Épicuriens avaient été ignoblement chassés de la cité de Lyctos et menacés, au cas où il leur prendrait fantaisie de revenir, d'un châtiment digne d'eux : ils seraient mis au carcan sur la place publique, enduits de lait et de miel pour attirer les mouches et les abeilles, ainsi transformés à leur corps défendant en la coupe de Memmius ou quelque bouche mantique ${ }^{29}$ !

Un des combats les plus constants des Épicuriens les opposa à l'ensemble du polythéisme grec et aux autres sectes sur le chapitre de la divination. Le déterminisme heurtait une physiologie fondée sur des çombinaisons d'atomes aléatoires et sur la déviation spontanée d'un clinamen; la providence

24 F. Longo Auricchio, A. Tepedino Guerra, "Aspetti e problemi della dissidenza epicurea", CErc 111 (1981), p. 25-40; D. SEdLEY, "Philosophical Allegiance in the GrecoRoman World", in M. Griffin, J. Barnes (éds), Pbilosopbia togata. Essays on Pbilosophy and Roman Society, Oxford, 1989, p. 97-119.

25 Cf. P. BOYANCÉ, Lucrèce et l'épicurisme, Paris, 1963, chap. III.

26 Philod, De dis III, col. 14, Pap. 157/152 Diels. Même le Barbare Apollonios de Tyane, étant un homme divin, a dès l'enfance parlé un pur attique (Philstr., V. $A p .1,7$ ).

27 Cf. par ex. Sophiste, 235d sq.

28 Élien, fr, 42a Domingo-Forasté.

29 Le miel en divination ou en poésie : cf. les Thries (Hymn. bom. à Hermès), Aristée, etc. (W.H. Roscher, Nektar und Ambrosia, 1883, p. 69 sq.; R. Triomphe, Le lion, la vierge et le miel, Paris, 1989, p. 255 sq.). 
ruinait l'ataraxie divine. Aussi les Épicuriens refusaient-ils d'admettre les oracles et la providence ${ }^{30}$, ce qui, malgré leur participation aux sacrifices et aux sacerdoces ${ }^{31}$, les excluait de facto du consensus polythéiste. De ce point de vue, un Épicure d'allure oraculaire est effectivement choquant. Le fait que le buste de Copenhague ait été abîmé par la perforation plaide aussi pour une intervention anti-épicurienne, destinée à tourner en dérision les fondements de la secte, à l'image du châtiment promis aux Épicuriens de Lyctos. Mais il s'agirait bien alors d'une caricature, non d'une inversion. Lucrèce lui-même a prêté à Épicure une majesté de prophète ${ }^{32}$. Épicure est un autre Asklépios dont les apparitions rêvées apportent la guérison. Nouvel Héraklès, il a débarrassé l'humanité des monstres qui l'épouvantaient ${ }^{33}$. On pouvait donc le railler à ce titre, simplement en prenant la secte à ses propres mots.

Mais inversement, pourquoi ses thuriféraires, dans leur zèle missionnaire, n'auraient-ils pas emprunté aux mours du temps le procédé de la tête parlante, qu'ils pensaient utiliser sans tromper personne parmi les leurs? D'autant qu'il n'est pas sûr que de tels procédés aient ordinairement trompé les Grecs, ni que ce bon sens ait nui à leur efficacité religieuse. Ou bien on goûte l'illusionnisme comme spectacle, ou bien, si l'on est enclin aux rencontres surnaturelles, on n'a pas besoin de ces artifices ${ }^{34}$. Les prodiges narrés par Ælius Aristide, ce névropathe mystique, sont de simples coïncidences ${ }^{35}$, non des trucages.

Aller plus loin dans nos hypothèses suppose une prise en compte plus précise des réalités religieuses des premiers siècles de notre ère : comment les Épicuriens s'y inscrivaient-ils tout en s'en démarquant pour affirmer leur identité propre?

Parmi les exemples de statues truquées fournis par Poulsen, un seul entre en relation avec l'épicurisme, tout en offrant le plus proche parallèle avec le buste de Copenhague : il s'agit précisément de ce Glykon (le doux, le sucré) fabriqué de toutes pièces par Alexandre Pseudomantis, dans le récit du

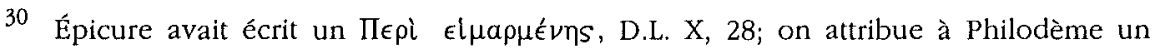
ouvrage sur la providence: M. Ferrario, "Filodemo 'Sulla provvidenza'? (PHerc. 1670)", CErC 2 (1972), p. 67-94. Cf. DIOG. CEN., fr. 53-54 Smith et en dernier lieu M.F. SMiTH, "Excavations at Oinoanda 1997: the new Epicurean texts", AS 48 (1998), p. 125-170.

31 L'Épicurien Hermon, du Banquet de Lucien, est un prêtre des Dioscures, issu d'une grande famille. Lépidus, haut personnage nommé dans Alexandre, était grandprêtre du culte d'Auguste et de Rome, cf. infra, n. 50.

32 LUCR., I, 62-79; III, 1-30; V, 1-54.

33 Lucr., I, 62-79 et V, 22-51. Cf. J. Pigeaud, "Quel dieu est Épicure ?", REL 50 (1972), p. 139-162.

34 Sur ces trucages : $c f$. R. Demangel, "Variations sur la pistis", RIDA 2 (1949) (Mélanges F. De Visscher I), p. 223-241.

35 par ex. Arsto., Disc. III, 45. 
même nom de Lucien. Le satiriste n'a pas de mots trop durs pour dénoncer les artifices du charlatan, la fausse tête de toile qui paraissait coiffer les replis d'un gros serpent inoffensif enroulé autour du cou d'Alexandre, les crins qui permettaient d'en actionner la gueule pour qu'elle parût s'ouvrir et se fermer, le demi-jour favorable à ses épiphanies dans une pièce étroite où l'on n'était pas plus tôt entré que déjà on était poussé vers la sortie, etc. ( $(12$ et $15-16)$. Le clou de ces séances oraculaires, réservé aux clients les plus riches ou les plus généreux, consistait en des consultations directes du dieu qui répondait de vive voix aux questions posées. Ces oracles, appelés «autophones », étaient obtenus par le moyen d'un tuyau fait de trachées de grues emboîtées : l'une des extrémités du tuyau était insérée dans la gueule du « dieu », et l'autre, invisible des consultants, passait dans une pièce voisine où un compère proférait les réponses dans l'embouchure $(\$ 26)$. Or l'Alexandre de Lucien tenait les Épicuriens pour les pires ennemis de son industrie oraculaire. Il les associait même aux Chrétiens dans sa détestation et, lors des mystères qu'il instaura, les impurs exclus lors de la proclamation inaugurale étaient précisément les Épicuriens et les Chrétiens, définis par leur commun «athéisme ${ }^{36}$. Alexandre scandait «Dehors les Chrétiens!», et la foule lançait ce répons : «Dehors les Épicuriens ! » $(\$ 38)^{37}$.

En quoi les Épicuriens étaient-ils si odieux à Alexandre ? Combattaient-ils simplement ses stratagèmes? Suffira-t-il d'opposer la raison à la superstition pour rendre compte de cette inimitié $e^{38}$ ?

36 Sur l'athéisme supposé d'Épicure, $c f$. D. Oввілк, "The atheism of Epicurus", GRBS 30/2 (1989), p. 187-223; G. Giannantoni, "Epicuro e l'ateismo antico", in G. Giannantoni, M. Gigante (éds), Epicureismo greco e romano. Atti del congresso intemazionale (Napoli, 19-26 maggio 1993), Centro di studio antico, Napoli, Bibliopolis, 1996, p. 21-63.

37 Sur les rapports entre épicurisme et christianisme $c f$. W. Schmid, Epicuro e l'epicureismo cristiano, trad. it., Brescia, 1984; A. J. Malmerbe, "Self-definition among Epicurean and Cynics", in B.F. MEYer et E.P. SANDERs (éds), Jewish and Christian SelfDefinition, Londres, 1982, p. 46-59. Sur l'organisation des Chrétiens, entre autonomie locale sous la surveillance des évêques, et direction épistolaire, $c f$. A. Harnack, Die Mission und Ausbreitung des Christentums in den ersten drei Jabrbunderten, Leipzig, 1924, I, p. 382-386 : les Chrétiens du Pont quant à eux sont assez nombreux en 190 pour bénéficier de plusieurs évêques (p. 383). Sur la direction de conscience chez les Épicuriens, cf. supra n. 9.

38 C'est l'opinion générale des commentateurs : cf. U. VICTOR, Lukian von Samosata. Alexandros oder der Lügenprophet, Leiden, 1997 (Religions in the Graeco-Roman World, 132); C.P. Jones, Culture and Society in Lucian, Harvard U.P., Cambridge, Mass., London, Engl., 1986; M. CASTER, Éludes sur Alexandre ou le faux prophète de Lucien, Paris, 1938; id., Lucien et la pensée religieuse de son temps, Paris, 1937; M. Croiset, Essai sur la vie et les ceuvres de Lucien, Paris, 1882. Jones (p. 45) donne une pleine valeur militante (et non de simple emprunt à la comédie) au scepticisme de Lucien, qui ne s'attaque pas à des cultes en déclin, mais aux plus florissants d'entre eux. Il note p. 24 que Lucien fait souvent des philosophes les représentants ou les défenseurs des croyances religieuses et des pratiques magiques qu'il ridiculise. 
On sait que le récit très partial de Lucien masque un phénomène religieux qui survécut d'un siècle et demi à son fondateur et toucha quasi tout l'Orient méditerranéen, attirant même de hauts dignitaires romains ${ }^{39}$. Se donnant pour un descendant mystique d'Asklépios et un amant de la Lune, Alexandre émerge dans l'histoire des religions comme un étonnant médiateur entre l'ancienne religion (incubation thérapeutique et tradition oraculaire; pythagorisme et mystique éleusinienne) et une religion nouvelle où le dieu, lui-même simple émanation du divin suprême, serait réellement présent sur le théâtre de son culte, incarné par un animal et représenté par son prophète, son envoyé, son découvreur, le fondateur de son culte. Certains passages de Lucien font presque écho à un message chrétien, tout en s'expliquant plutôt par la doctrine du Timée ou des Stoïciens ${ }^{40}$. Ainsi on demande si Alexandre est une réincarnation de Pythagore. Réponse de l'oracle :

L'âme de Pythagore tantôt meurt et tantôt reprend force.

Mais celle du Prophète est une effusion de l'esprit divin ( $\delta$ lı $\rceil s$ $\phi \rho \epsilon \nu o ́ s$

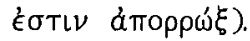

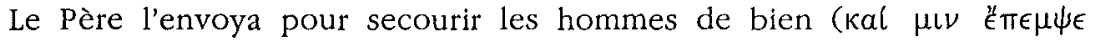

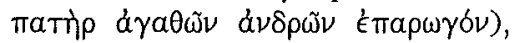

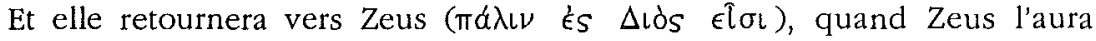
frappée de sa foudre $(\$ 40)$.

Des accointances affirmées avec le christianisme apparaissent chez cette autre bête noire de Lucien, Pérégrinus, alias Protée, qui suscita un oracle bien plus modeste (une image miraculeuse dans sa cité natale de Parion) ${ }^{41}$ : le Cynique Pérégrinus, dit Lucien, après bien des tribulations dont un séjour en Palestine et une adhésion temporaire à la secte chrétienne, se donna pour un nouvel Héraklès ou un autre Empédocle ${ }^{42}$, et s'immola par le feu en grand spectacle, à Olympie, lors des Jeux de 165, devant un fort concours de peuple qui s'empressa de témoigner de son apothéose et de ses réapparitions.

L'Alexandre réel n'était donc pas un mystificateur isolé, mais un phénomène de société que Lucien combat au nom d'un parti pris. Or ce combat n'est pas celui de la raison contre l'obscurantisme. Selon Lucien le prophète prend certes bien soin de lancer son oracle attrape-nigaud auprès des épais Paphlagoniens. Aurait-il eu moins de succès auprès des gens cultivés ? Un grand personnage romain comme Rutilianus est son plus grand dévot. C'est à Olympie même, en pleine panégyrie, que Pérégrinus trouve ses dupes. Les philosophes sont chez Lucien grands amateurs d'histoires miraculeuses,

39 Cf. Victor, o.c. (n. 38), Introduction, et JoNes, o.c. (n. 38), p. 133-148.

to $C f$. par ex. D.L., VII, 147 (= SVF II, 1021) pour la conception stö̈cienne du dieu.

41 Lucien, Per. Cf. Athénagoras, Supplique XXVI, 3-4. Autres témoignages : Souda, s.v. Фıdóotpatos ó mpw̃tos; Gell., XII, 11, cf. VIII, 3; Philstr., Vit. Soph. XIII, 563; Lucien, Démonax, 3; Tert, Mart. IV, 625; Tatien, Adv. Graec. XXV, 264; Amm., XXIX, 1, 39.

42 Autre modèle possible : le brahmane Kalanos. Cf. PLut., Alex, 8; 65; et surtout 69; ARrien, Anab. VII, 3, 1-6. 
comme chez Philostrate les étudiants d'Athènes sont les premiers à saluer en Apollonius l'homme divin, quand il débarque au Pirée ${ }^{43}$. Et Lucien en personne nous décrit avec admiration, en pastichant certes Hérodote, les merveilles du sanctuaire d'Hiérapolis, dont quelques statues animées ${ }^{44}$.

Consulter un oracle n'implique pas qu'on ignore les procédés de ses desservants : la conscience plus ou moins claire du trucage n'a jamais empêché d'applaudir les prestidigitateurs. Lucien n'apprend rien à ses lecteurs en dénonçant la tête articulée du serpent Glycon ou les supercheries d'Alexandre. Mais il dénonce le procédé par refus partisan d'entrer dans ce jeu-là, exactement comme feront les Chrétiens, qui sont pourtant les derniers à sous-estimer la puissance des dieux païens ${ }^{45}$. Le consultant de l'oracle, lui, entre dans le jeu et, quels que soient les artifices de la mise en scène religieuse, sa démarche active l'engage émotionnellement et lui permet de donner un sens personnel aux réponses qu'il reçoit. Si Lucien, de son cồté, se place d'emblée hors-jeu, nous suggérons qu'il oppose une règle à une autre, une religion à une autre, et non la raison à la religion ${ }^{46}$. Lucien n'est pas un Voltaire. N'en déplaise à Frazer, quand la religion décline ce n'est pas la raison qui la remplace, mais une autre religion ${ }^{47}$.

Où se situe, en effet, le terrain de la guerre?

1. Lucien affiche dans l'Alexandre une appartenance explicite aux réseaux épicuriens, sur lesquels il projette une lumière assez vive ${ }^{48}$. On a minimisé cette appartenance au motif que Lucien était imperméable à la physique épicurienne : mais il a pu n'adhérer vraiment qu'au soir de sa vie, après n'avoir longtemps éprouvé pour la secte qu'une nette sympathie ${ }^{49}$; ou bien les controverses et les réseaux d'influence du temps l'ont amené à se ranger aux côtés des Épicuriens de manière à la fois informelle et plus militante qu'on ne croit. L'Alexandre évoque le cercle épicurien d'Amastris, autour d'un certain Lépidos qu'une inscription funéraire permet d'identifier

\footnotetext{
43 Philstr., V. Ap. IV, 17.

44 LUCIEN, Syria D., 32-37.

45 Cf. HIPp., Ref. IV; Eus., Prop. ev. V.
}

46 Le mensonge trouble la vie sereine (Tychiades dans Philops. 40). Et Lucien résiste à une mode plutôt qu'il ne combat pour les Lumières. Mais $c f$. Jones, o.c. (n. 38), chap. V, "Credulity and Fiction"

47 Cf. le finale du Rameau d'or (« Adieu à Némi »).

48 Sur l'orthodoxie et l'originalité de l'épicurisme au II $^{\mathrm{e}} \mathrm{s}$. d'après Diogène d'Enoanda : cf. M..F. SMITH, Diogenes of Oinoanda: The Epicurean Inscription, Naples, Bibliopolis, 1993 (introduction); D. CLAY, "A lost Epicurean Community", GRBS 30 (1989), p. 313-335; P. Gordon, Epicurus in Lycia. The Second-Century World of Diogenes of Oenoanda, Ann Arbor, Univ, of Michigan P., 1996, et vive critique de M.F. SMirH dans AncPbil 18 (1998), p. 216-220.

49 Cf. une conversion tardive de Minucius Felix au christianisme, après une longue adhésion à l'épicurisme, selon A.D. Simpson, "Epicureans, Christians, Atheists in the Second Century", TAPhA 72 (1941), p. 372-381. 


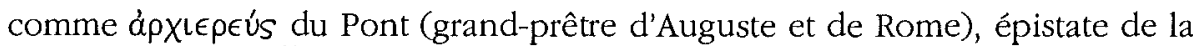
capitale Amastris ${ }^{50}$. Épicuriens et Chrétiens étaient si nombreux dans cette cité que jamais Alexandre ne voulut prophétiser pour ses habitants : il abominait la ville entière $(\$ 25)$. Lucien évoque aussi l'activité militante du cercle de Timocrate d'Héraclée, qui l'épaula dans son ébauche de procès contre Alexandre pour tentative d'homicide $(\$ 57)$. Le destinataire même de son récit est l'Épicurien Celse, le même peut-être que l'ennemi d'Origène, et avec lequel Lucien paraît s'unir dans la célébration exaltée d'Épicure ${ }^{51}$. Celse avait écrit un traité Contre la magie (Кarà $\mu a ́ \gamma \omega \nu, \$ 21$ ), et Lucien l'imite dans son engagement contre les sorcelleries d'Alexandre. Les cibles des Épicuriens varièrent en fonction des métamorphoses de la superstition dans leur environnement social ${ }^{52}$. C'était d'ailleurs une forme de ministère parmi d'autres, chez eux, que de combattre l'ennemi extérieur ${ }^{53}$ : des disciples plus physiologues se chargeaient des aspects proprement doctrinaux, mais chacun

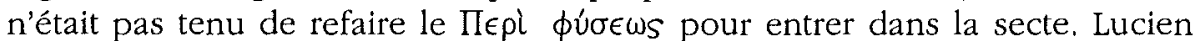
lui-même se montre fort brave contre Alexandre ${ }^{54}$ : il lui mord la main au lieu de la baiser $(\$ 55)$, ou bien guette ses bévues, les provoque par des questions pièges, pour les dénoncer publiquement $(\$ 53-54)^{55}$.

2. Alexandre est au contraire présenté comme un ennemi déclaré des Épicuriens : il va jusqu'à excommunier leurs adeptes ${ }^{56}$, à les faire lapider $(\$ 45)$, à peindre Épicure enchaîné dans la fange au fond des Enfers ${ }^{57}$, à organiser l'autodafé des Kuriai Doxai sur la place publique $(\$ 47)$ ! Cette inimitié est fondée sur la peur : "Car il avait, comme je l'ai dit, la plus grande peur d'Épicure : il voyait en lui un ennemi habile à contre-battre et à déjouer tous ses tours » ( $\$ 43)$. Peur, parce qu'Épicure connaît lés tours capables d'inverser

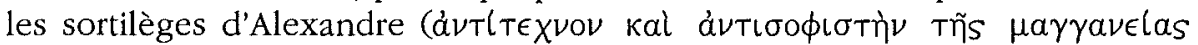
aủToũ). Les Épicuriens démontent ses supercheries (Celse dans son kaTd

50 CIG 4149: IGR III, 88; cf. VICTOR, o.c. (n.38), p. 151.

51 «Épicure, cet homme vraiment saint, ce génie vraiment divin, le seul qui ait connu le Bien dans sa réalité et en ait transmis la science, le libérateur de ceux qui ont écouté sa parole!» ( $(61$, trad. Caster).

52 Voir par ex. P.A. BRUNT, "Philosophy and Religion in the Late Republic", in M. Griffin, J. BARnes (éds), Philosophia togata. Essays on Pbilosophy and Roman Society, Oxford, 1989, p. 174-198; K. Summers, "Lucretius and the Epicurean Tradition of Piety", CPb 90 (1995), p. $32-57$.

53 D'ordinaire des philosophies rivales, $c f$. l'activité du Colotès attaqué par Plutarque.

54 Pas assez fou pourtant pour braver la lapidation (44-45).

55 Mais il a été sensible à la séduction d'Alexandre (55)!

56 Quand Alexandre répondait és kópakas, le consultant éconduit « n’était plus reçu sous aucun toit; nul ne partageait plus le feu et l'eau avec lui; il était pourchassé de pays en pays, comme impie, comme athée, comme Épicurien, - ce qui était la pire injure » (46).

57 «On lui demanda un jour: Que devient Épicure chez Hadès? Des chaînes de plomb aux pieds, répondit-il, il est assis dans la fange » (25). 
$\mu a ́ \gamma(\omega \nu)$, ou dressent des pièges pour le faire succomber ( $\$ 44-45)$, montent des dossiers contre lui (le procès avorté de Lucien). Ils connaissent les ressorts psychologiques utilisés par Alexandre, et ils savent le danger de ces manipulations pour le repos du corps et de l'âme. "Il eût vraiment fallu un Démocrite, voire Épicure lui-même, ou Métrodore, enfin quelqu'un de ces

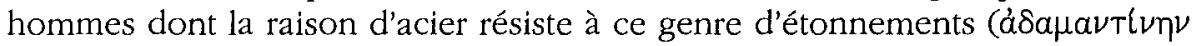

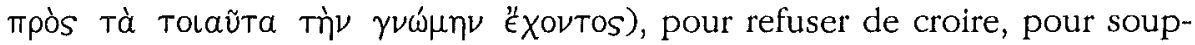
çonner la réalité, et, sinon pour découvrir le secret, du moins pour rester persuadé, a priori, que si le secret de cette sorcellerie lui demeurait caché, tout cela n'était pourtant qu'un mensonge, et ne pouvait pas exister (kai

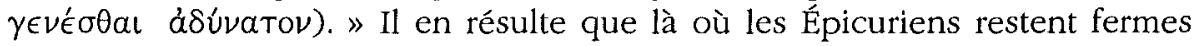
devant les sortilèges, Alexandre au contraire, croyant épouvanter autrui à son profit personnel, se rend lui-même victime d'un Épicure épouvantail! Ce

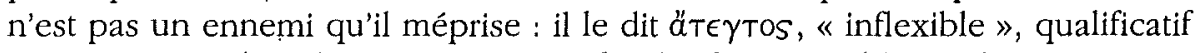
qui rejoint celui dont Lucien gratifie la fermeté d'âme des atomistes

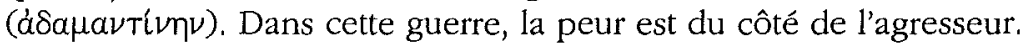

3. Les particularités de la réforme religieuse introduite par Alexandre ont été finement analysées par Ulrich Victor ${ }^{58}$ : le pari d'offrir un dieu réellement présent, visible, tangible, audible, au moins aux dates d'ouverture de l'oracle; un dieu qui exauce les prières à la lettre; un oracle qui s'engage à répondre à des questions non standardisées (comme font la plupart des oracles, endehors de Mallos)... Rien n'est plus opposé au Jardin : avoir installé le dieu parmi les hommes, alors que l'épicurisme l'exile dans les intermondes; lui donner forme animale, alors qu'Épicure lui assigne la forme humaine; lui prêter une écoute attentive, là où Épicure considère son indifférence comme constitutive de sa définition; avoir paré son héraut d'ornements barbares et fondé son culte sur un spectacle inouï, quand les Épicuriens, engagés dans les seuls cultes officiels et traditionnels, représentent leur fondateur sous les traits d'un citoyen idéal's?.

4. Mais en même temps il faut croire qu'Alexandre est en concurrence avec les Épicuriens: si Alexandre s'affiche avec une barpè, comme un descendant de Persée tueur de monstres, Épicure lui aussi a été présenté en tueur de monstres, qui décapite l'épouvantail de la religion ${ }^{60}$. Alexandre offre un culte guérisseur (un nouvel Asclépios), mais Épicure a lui aussi apporté des remedia à tous les maux de l'humanité. La douceur de Glycon répond aux délices partagés de l'épicurisme. Épicure fut un fondateur de secte et révéla l'image divine véritable : Alexandre ne prétend pas faire autre chose. La médiation qu'Alexandre introduit entre les dieux et les hommes, Épicure d'une autre façon l'a introduite cinq siècles plus tôt, en se donnant comme celui qui au milieu des hommes donne visage à l'ataraxie divine et y offre

\footnotetext{
58 VICTOR, o.c. (n. 38).

59 Cf. ZANKER, o.c. (n. 4), p. 113 sq.

60 LUCR, I, 62-79.
} 
accès, Épicure et Alexandre ont tous deux pris en charge la tradition religieuse des Grecs, pour la réformer et la réorienter : ils n'ont pas introduit de dieux étrangers, mais simplement un nouveau médiateur. Enfin l'épicurisme se propageait par des libelles comparables aux épîtres pauliniennes : or l'accent mis sur les questions et les réponses écrites de l'oracle d'Abonotique, les citations qu'en fait Lucien, suggèrent qu'à cette époque où Claros diffusait des oracles théologiques ${ }^{61}$, des recueils circulaient et tendaient à appuyer la théophanie de Glycon sur des révélations écrites qui en faisaient aussi une sorte de religion du livre embryonnaire, à comparer avec celle de Julien le Théurge ${ }^{62}$ : ici oracles et philosophie font déjà aussi bon ménage que chez Porphyre, et on les trouvera encore combattus ensemble dans les Pbilosophoumena d'Hippolyte. À CEnoanda où par son portique inscrit l'Épicurien Diogène s'offre comme un sauveur, un oracle théologique de Claros à peu près contemporain, inséré au-dessus d'une porte dans le mur d'enceinte (et peut-être destiné à protéger la cité d'un fléau comme la peste qui incita l'oracle d'Alexandre à faire aux particuliers une recommandation du même ordre ${ }^{63}$ ?), concurrence la médecine de Diogène avec des remèdes (une définition du Dieu dont les dieux traditionnels ne sont que les « anges », les envoyés; un rite de prière) qui, pour n'être pas contradictoires avec ceux d'Alexandre, n'en auraient pas moins, cette fois, presque pu convenir à un Chrétien aussi bien qu'à un bénéficiaire éclairé de la paideia ${ }^{64}$. Nous sommes apparemment en une période charnière, où les identités s'aiguisent dans la controverse, où la philosophie devient l'appui argumentatif et la forme la plus engagée des revendications religieuses.

5. Résumant tout le reste, opposition et rivalité, le danger que la religion d'Alexandre fait courir aux hommes, en les détournant du salut épicurien et en annexant une certaine philosophie ${ }^{65}$, suffirait à faire d'elle la pire incarnation de l'épouvantail religieux que les Épicuriens combattent. En effet, Alexandre fonde toute son entreprise, Lucien y insiste, sur l'espoir et la peur, c'est-à-dire précisément les deux passions qui ruinent la paix de l'âme, et qu'une fausse religion génère en suscitant l'espoir des récompenses et la crainte des châtiments. Comment y parvient-il ? Au-delà de l'abus du specta-

61 K. BURESH, Klaros, Untersuchungen zum Orakelıesen des späteren Altertums, Leipzig, 1889; L. RoBert, "Trois oracles de la Théosophie et un prophète d'Apollon", CRAI (1968), p. 568-599.

62 Cf. l'ouvrage classique de H. LEwy, Chaldaean Oracles and Theurgy. Mysticism, Magic and Platonism in the later Roman Empire, Paris, $1978^{3}$ [1956].

63 LuCien, Al., 36.

64 L. RoberT, "Un oracle gravé à Oinoanda", CRAI (1971), p. 597-619 (p. 614); S. Mitchell, Anatolia, Oxford, 1993, II, p. 44, 48. LACTANCE, Inst. div. 1, 7 prit l'oracle à son compte moyennant un léger correctif.

65 Là encore, l'oracle d'Alexandre a partie liée avec la gnose et la thérgie, où la philosophie se mêle à une mystique ésotérique mêlée de pratiques magiques, et qui sont appuyées sur l'allégorèse dont les Stoïciens, amis d'Alexandre, les Pythagoriciens, et les disciples de Platon, ont fait un usage systématique. 
culaire, son vrai tort est de faire croire que la divinité qu'il exhibe en théophanie n'est pas une image produite par l'homme, mais le dieu en personne. Il introduit une pernicieuse confusion entre les ä $\delta \eta \lambda \alpha$, auxquels le raisonnement accède appuyé sur les signes (atomes, vide, infini, et sans doute dieux des intermondes) ${ }^{66}$, et les choses qui sont $\dot{\epsilon} v a \rho \gamma \eta \tilde{n}$, évidentes et tombant sous les sens (ainsi la prolepse des dieux, Lettre à Ménécée, 123). Quand les Épicuriens vivent dans la paix quelque peu narcissique d'un dialogue avec l'image, Alexandre précipite ses victimes dans l'abîme de l'être.

Le buste parlant d'Épicure a pu offrir les plaisirs d'une illusion utile; le serpent autophone d'Alexandre brouille tous les repères par sa seule présence réelle.

EPHE, Sciences religieuses

Renée PietTrke

Paris, Sorbonne 